\title{
DESTRUCTION OF ANTIFERROMAGNETIC ORDER BY LOCALIZED POLARONS
}

\author{
Z. Wilamowski and E.M. HankIEwicz \\ Institute of Physics, Polish Academy of Sciences \\ Al. Lotników 32/46, 02-668 Warszawa, Poland
}

\begin{abstract}
Analysis of a finite spin cluster indicates a possibility of the formation of purely magnetic polarons in low-dimensional antiferromagnets. It is shown that for parameters characteristic of $\mathrm{CuO}_{2}$ based superconductors a strong antiferromagnetic polaron can occur. The destruction of antiferromagnetic order by the presence of a magnetic polaron is discussed.
\end{abstract}

PACS numbers: 74.20.-z, 75.10.-b, 74.20.Mn

The concept of a magnetic polaron as a possible mechanism of the formation of the Cooper pair in high- $T_{\mathrm{c}}$ materials was discussed by several authors $[1,2]$, but none of the models was really convincing. The idea of Mott polaron [1] is of pure classical character so it cannot effectively describe the antiferromagnetic (AF) polaron. Pines [2] introduces a phenomenological parameter which stands for quantum fluctuations. He convincingly discusses their role in spin dynamics but his model is limited to linear response, while the total strength of $p-d$ coupling in $\mathrm{CuO}_{2}$ planes, $N_{0} \alpha=\sum_{i} J_{p-d}(i)$ [3], i.e., sum of exchange constants, $J_{p-d}$, of $p$-electron with localized $d$ spins, is a few times larger than the exchange coupling, $J_{d-d}$, between $\mathrm{Cu}$ spins. In this paper we argue that to clarify this concept much more careful modeling of the formation of AF magnetic polarons has to be performed. Our new results [3] show that a really effective binding of AF polarons in Cooper-like pairs can take place when (i) the spatial distribution of the electronic state is of "comb-like" shape, i.e., when the effective $p-d$ field acts on every second $\mathrm{Cu}$ spin, (ii) when the size of the AF cluster, or the range of the AF coherence is small enough (the energy of pair formation is inversely proportional to the coherence range). The most important features of these calculations are a rigorous treatment of the quantum fluctuations and an analysis of the data also in the limit of strong $p-d$ coupling.

We show that the polarons, which are formed as a wave packet of the electronic states from the bottom of the electronic band, destroy the AF order allowing formation of "comb-like" bipolarons. Our method [3] allows us to consider a relatively big cluster, of the order of 20 coupled spins, in a rigorous way. For 2D clusters our method is more limited, but some of the effects, i.e., the formation of a strong magnetic polaron can be also studied. 
In Fig. 1 the effective susceptibility, i.e., the energy gain due to polaron formation, $\Delta E_{\text {ex }}$, normalized by the sum of the squares of $J_{p-d}(i)$, is plotted as a function of the total strength of $p-d$ coupling, $N_{0} \alpha$. The numerical results are very different from the classical value and they are strongly dependent on the polaron shape (distribution of $J_{p-d}(i)$ on local spins). The variety of the results reflects different types of AF magnetization. The classical limit corresponding to a homogeneous susceptibility is achieved for a smoothly shaped polaron at the limit of very strong $p-d$ exchange only. For a weak $p-d$ coupling the homogeneous polaron does not couple to an AF cluster, but at stronger coupling $\left(N_{0} \alpha \approx 9 J_{d-d}\right)$ a break of the AF order, induction of a magnetic moment, and an energy gain occurs. When the non-homogeneity of the polaron shape increases the susceptibility increases. The susceptibility gets its maximum for $N_{0} \alpha$ of the order of few $J_{d-d}$. Generally, the increase in the susceptibility with $N_{0} \alpha$ indicates the importance of the nonlinear effect and the failure of linear response models.

The dependence of the energy of the polaron formation on the size of the polaron (represented by the variance of the Gaussian distribution, $\sigma$ ), for a fixed value of $p-d$ exchange, is shown in the inset in Fig. 1. In the limit of a smooth shape, i.e., for large $\sigma$, the polaron energy decreases with $1 / \sigma$. Since the kinetic energy increases with the polaron localization as $1 / \sigma^{2}$, a minimum of the total energy, corresponding to the formation of a weak magnetic polaron, occurs. For

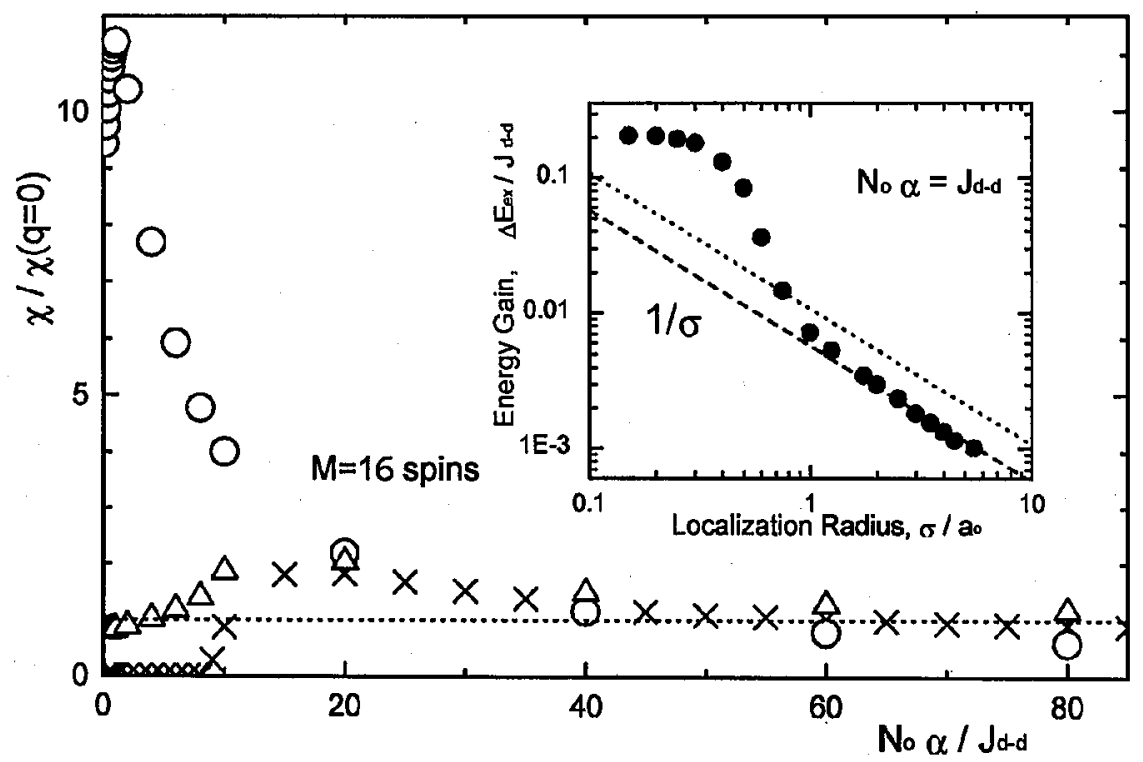

Fig. 1. The effective susceptibility vs. the strength of $p-d$ exchange, $N_{0} \alpha$ normalized by $d-d$ coupling $J_{d-d}$. The susceptibility in units of classical value $\chi$ at $q=0$. Various sets stand for various types of magnetic polarons: strong polaron $\left(\sigma=0.5 a_{0}-\bigcirc\right)$, weak $\left(\sigma=2.6 a_{0}-\Delta\right)$, and homogeneous electron distribution $(x)$. The inset shows the dependence of the $p-d$ energy as a function of the polaron size, $\sigma$. The dashed line corresponds to smooth polaron, the dotted one - to the classical approach. 
the parameters characteristic of high- $T_{\mathrm{c}}$ superconductors $\left(N_{0} \alpha=0.7 \mathrm{eV}, J_{d-d}=\right.$ $75 \mathrm{meV}$, and $m^{*}=m_{0}$ ) the expected localization of such a polaron is of the order of $\sigma \approx 1.5 a_{0}$ and the corresponding energy is $25 \mathrm{meV}$. But for a stronger localization, an extra energy gain would appear (see inset in Fig. 1). The gain is by an order of magnitude greater than the value obtained from smooth polaron extrapolation. Such a dependence indicates the possibility of an extra localization. The numerically evaluated minimum of the total energy shows that the polaron has rather properties of a strong polaron. Its localization is expected to be $\sigma \approx 0.5 a_{0}$ and the energy of about $100 \mathrm{meV}$. This value is much bigger as compared to a classical polaron caused by the dielectric lattice polarization.

The analysis of the problem of formation of a magnetic polaron in a $2 \mathrm{D}$ AF lattice leads to similar conclusions. In particular, the energy gain due to the formation of a strongly localized polaron is of the same order of magnitude.

The formation of a magnetic polaron is accompanied with a change of the AF correlation radius, $\rho_{\mathrm{c}}$. In Fig. $2 \mathrm{a} \rho_{\mathrm{c}}$ is plotted as a function of $p-d$ exchange strength, $N_{0} \alpha$. The dependencies $\rho_{\mathrm{c}}\left(N_{0} \alpha\right)$ are different for various polaron shapes. For a homogeneous distribution of the $p-d$ field over the AF cluster the dependence has a step-like character, which corresponds to the break of AF order. In
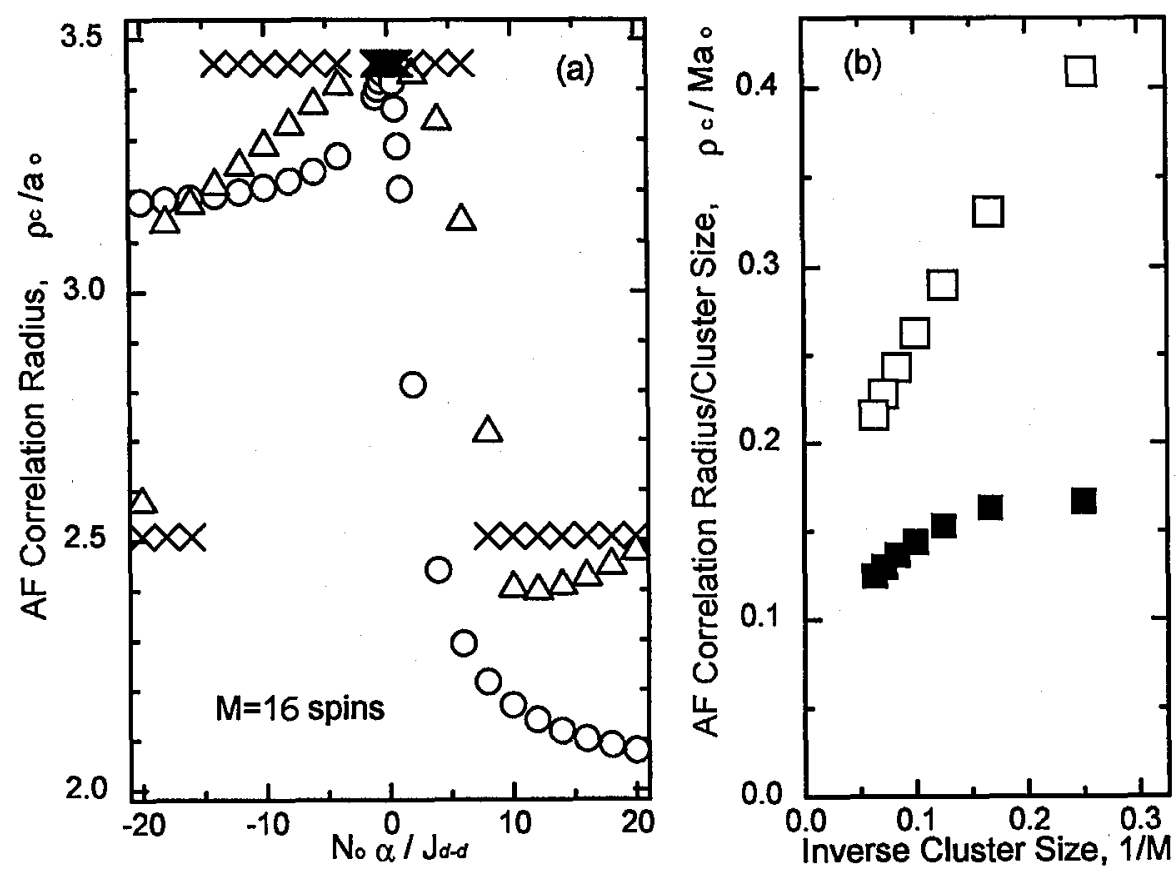

Fig. 2. (a) The correlation radius as a function of the strength of $p-d$ coupling $N_{0} \alpha$ for various magnetic polarons: strong polaron $\left(\sigma=0.5 a_{0}-O\right)$, weak $\left(\sigma=2.6 a_{0}-\Delta\right)$, and homogeneous electron distribution $(x)$; (b) the correlation radius normalized by the size of cluster $M$ for a non-perturbed AF cluster (open symbols) and for a cluster affected by a strong magnetic polaron (solid) are plotted as a function of the inverse of the cluster size. 
other cases the correlation radius decreases with the square of $N_{0} \alpha$ for a weak $p-d$ strength, then it saturates at $N_{0} \alpha>10 J_{d-d}$. The decrease in $\rho_{\mathrm{c}}$ is more pronounced for a more localized polaron, but the saturation value is similar for different types of polarons. In Fig. $2 \mathrm{~b}$ the correlation radius (normalized by the length of the AF chain, $M a_{0}$ ) for an unaffected AF cluster and for the cluster with a strongly coupled $\left(N_{0} \alpha \gg J_{d-d}\right)$ polaron are plotted as a function of the inverse of the cluster size, $M$. The decrease in the correlation radius by the factor 2 corresponds to the real cutting of the chain into two uncoupled parts, or, what is equivalent, to the presence of a non-magnetic impurity in the AF chain. As it is shown in Fig. 2b, the effect of the strong polaron is close to the real cutting of $\mathrm{AF}$ chain. One could expect that a presence of a number of polarons, $N_{p}$, at a long AF chain of $M$ spins leads to the resulting correlation radius $\rho_{\mathrm{c}} \approx a_{0} / x$ where $x=N_{p} / M$.

The same formalism stands for the $2 \mathrm{D}$ case, but the calculation of the correlation range, $\rho_{\mathrm{c}}$, and its extrapolation to a big system, is characterized by a much bigger numerical error. Nevertheless, the comparison of correlation radii for a non-perturbed cluster, a cluster "doped" with a spinless site, and a cluster affected by a polaron leads to the conclusion that the destruction efficiency for sufficiently big strength, becomes equivalent to that of non-magnetic doping in a 2D AF lattice.

The effect of destruction depends on the value and the sign of $p-d$ coupling, $N_{0} \alpha$ (see Fig. 2a). The destruction by an AF $p-d$ coupling (positive) is much more effective. One of the reasons is that the AF $p-d$ coupling of a strong magnetic polaron can be treated as a compensation of a local spin $d$. Full analysis of the dependence of destruction effects on the polaron shape and $N_{0} \alpha$ is very complex. In addition to the compensation effect, one has to consider the tendency to induce a local staggered magnetization by the strongly non-homogeneous exchange field, $J_{p-d}(i)$, and the break of AF coupling by the strong homogeneous fields.

The results show that spin interactions alone lead to the carrier localization and the formation of strong magnetic polarons. The energy of the polaron formation is enhanced by the electric lattice polarization, which is not considered in this paper. Generally, however, the polaron should be treated as an object with specific spatial distribution of the electronic charge, magnetization of localized spins, and electric lattice polarization. The type of polaron discussed in this paper is expected to be responsible for the destruction of the $\mathrm{AF}$ order in under-doped $\mathrm{CuO}_{2}$-based superconductors.

\section{Acknowledgments}

This work is supported by the Committee for Scientific Research grant No. 2 P03B 000716.

\section{References}

[1] For review see: A.S. Alexandrov, N. Mott, High Temperature Superconductors and Other Superfuids, Taylor and Francis, London 1994.

[2] D. Pines, in: The Gap Symmetry and Fluctuations in High-T $T_{\mathrm{c}}$ Superconductors, Eds. G. Deutscher, D. Pavuna, S.A. Wolf, Plenum Press, New York 1998, p. 111.

[3] E.M. Hankiewicz, R. Buczko, Z. Wilamowski, Acta Phys. Pol. A 97, 185 (2000). 